\title{
Research of a new method of sealing of sandy soil foundations
}

\author{
Oleg Minaev ${ }^{1, *}$ and Michail Kolosov ${ }^{1}$ \\ ${ }^{1}$ Admiral Makarov State University of Maritime and Inland Shipping, 5/7, Dvinskaya str, Saint- \\ Petersburg, 198035, Russia
}

\begin{abstract}
The paper presents the results of the author's research aimed at finding new methods of vibrodynamic sealing of sandy soils foundation by using the energy of water flow. The data of water flow parameters providing the given speed of its movement without the significant carryover of sand particles based on the theoretical studies, as well as the laboratory studies of sandy soil dumping into a water flow (with an average horizontal velocity of about $10-15 \mathrm{~cm} / \mathrm{s}$ ) on a specially designed hydraulic tray are described in the paper.
\end{abstract}

\section{Introduction}

The "island type" oil platforms are made in the form of an inverted cup and usually have a diameter exceeding $100 \mathrm{~m}$, with an opening filled with inwash sand.

The analysis of the condition of the water-saturated sandy soils of the foundations and structures made by free inwashing and underwater dumping in the absence of current shows that regardless of the granulometric composition, they are settled with a skeleton density of a fluffy consistency and require compaction, especially in earthquake zones.

Today, vibrodynamic soil compression techniques for structures and foundations are well-known and used worldwide [1-13].

To compact the core sand of the "island type" ice-resistant stationary platform at the offshore oil and gas condensate field Chaivo-1 (Ice-resistant stationary platform (ISP) "Chaivo-1"), the author proposed an explosive method for compacting the sand core of the oil platform. According to the project variant of an oil platform of this type developed in Russia, performed in the form of a multi-faceted metal shell, the explosive method of compaction was defined as the main one. However, in view of the very tight deadlines of platform construction during the summer period in the Northern climatic conditions, in particular, on Sakhalin Island (Russia), the author conducted special research studies aimed at reducing the construction time by combining the operations of inwashing and soil compaction. The study was conducted at the request of the "SakhalinNIPImorneft" Institute for the general contractor "Sakhalinmorneftemontazh" of the "Sakhalinmorneftegaz" production association.

\footnotetext{
* Corresponding author: minaev.op@bk.ru
} 
However, the author of the article argues that the search for fundamentally new ways of compression, in particular, with the use of kinematic energy of water flow for vibrodynamic foundation soil compression, is of great importance.

It is also known [14-16] that there is the experience of free deposition of sandy soils into horizontally flowing water at banquet-free method of river closure. There is no significant increase in density in this case.

The available data concerning soil settling during river closure do not provide enough information about the impact of the fixed horizontal flow on sandy soil deposition mainly due to a significant carryover of smaller particles.

Taking this into account, the author of the article set the task of looking into the possibility of increasing sandy soil density during dumping and hydraulic deposition of soil into a water flow with the specified parameters without the significant particles carryover.

The article presents the methodology and results of the study conducted on a specially developed experimental stand which, for the first time, allowed receiving data on the increase of density of sand depositing in a moving water flow with the preset design parameters of movement.

The developed project of metal shell of the "island type" ISP, was given by the general project organization "Melnikov Central Research and Design Institute of Steel Structures " to the investor in the United States (Houston) to choose from alternative project options for implementation, including on similar deposit fields in the north of Sakhalin Island and other places.

\section{Calculations}

The momentum of the jets can be found by the formula [17] as in equation (1):

$$
K_{j e t}=\rho_{W} \cdot Q \cdot V_{\text {hole }},
$$

where $\rho_{\mathrm{w}}, \mathrm{Q}, \mathrm{V}_{\text {hole }}$ - density of water, water flow velocity and water flow rate immediately at the outlets, respectively.

Assuming that the momentum is equal to the reaction of interaction of outflowing jets and water volume in the tank we calculate as in equation (2):

$$
K_{\text {jet }}=P_{\text {react }}=\rho_{w} \cdot V \cdot a=\rho_{w} \cdot \mathrm{V} \cdot \frac{\mathrm{u}_{\max }}{2 \pi R} \cdot \frac{1}{R} \int_{0}^{R} u_{\max } \frac{\mathrm{r}}{\mathrm{R}} \mathrm{dr}=\rho_{\mathrm{w}} \cdot \mathrm{V} \cdot \frac{\mathrm{u}_{\max }}{2 \pi \mathrm{R}} \cdot \frac{u_{\max }}{2},
$$

where $\mathrm{V}$ and $\mathrm{R}$ are radius and volume of the tank, $\mathrm{r}$ is the distance from the center of the tank along the radius.

Equating the expressions for the momentum of jets and water volume of in the tank we have as in equation (3):

$$
\rho_{\mathrm{w}} \cdot \mathrm{Q} \cdot \mathrm{v}_{\mathrm{hole}}=\rho_{\mathrm{w}} \cdot \pi \cdot \mathrm{R}^{2} \cdot \mathrm{h} \cdot \frac{\mathrm{u}_{\text {max }}^{2}}{4 \pi \mathrm{R}}
$$

or transforming as in equation (4)

$$
\mathrm{Q} \cdot \mathrm{v}_{\mathrm{hole}}=\mathrm{R} \cdot \mathrm{h} \cdot \frac{\mathrm{u}_{\text {max }}^{2}}{4}
$$


where $\mathrm{h}$ is the height of the tank.

From equation (4) we determine the maximum linear velocity of water low in the tank:

$$
u_{\max }=2 \sqrt{\frac{\mathrm{Q} \cdot \mathrm{V}_{\mathrm{hole}}}{\mathrm{h} \cdot \mathrm{R}}}=2 \sqrt{\frac{705 \mathrm{~cm}^{3} \cdot 75 \mathrm{~cm} / \mathrm{s}}{31,25 \mathrm{~cm} \cdot 26,8 \mathrm{~cm}}} \approx 16 \mathrm{~cm} / \mathrm{s} .
$$

with

$$
V_{\text {hole }}=\frac{Q \cdot 4}{48 \pi \cdot d_{\text {hole }}^{2}}=\frac{705 \mathrm{~cm}^{3} / \mathrm{s} \cdot 4}{48 \cdot \pi \cdot(0,5 \mathrm{~cm})^{2}}=75 \mathrm{~cm} / \mathrm{s},
$$

where $d_{\text {hole }}$ - is the diameter of the holes in the flow pipe.

Given the theoretical calculations of uniform velocity distribution over the height of the cylinder and uniform velocity changes along the radius from 0 in the center to $u_{\max }$ at the distance $\mathrm{R}$, we can assume that the real velocities in the tank will be different from those calculated. This discrepancy can be evaluated by experimental data measurements.

Assuming that particle carryover from the tank will be mainly due to vertical velocities. $\mathrm{u}_{\text {vert }}$ of water flow from the tank, we find as in equation (7):

$$
\mathrm{u}_{\text {vert }}=\frac{\mathrm{Q}}{\pi \cdot \mathrm{R}^{2}}=\frac{705 \mathrm{~cm}^{3} / \mathrm{s}}{\pi \cdot(26,8 \mathrm{~cm})^{2}}=0,3 \mathrm{~cm} / \mathrm{s}
$$

From [17] we determine the maximum diameter of the entrained particles as $\mathrm{d}=0,07 \mathrm{~mm}$ for the hydraulic size $\mathrm{W}_{\mathrm{o}}=3 \mathrm{~mm} / \mathrm{s}$. As a rule, all particles whose $\mathrm{W}_{\mathrm{o}}<\mathrm{u}_{\text {vert }}$ should be carried away by the upflow out of the tank. Typically, the percentage of particles $\mathrm{d}<0,07 \mathrm{~mm}$ in the sands of medium size is small, so it can be expected that the particles carryover will be negligible in the experiments.

\section{Experiments}

The laboratory setup consisted of a hydraulic tray with the casing of $0.6 \times 0.59 \mathrm{~m}$ and the side walls and the bottom with the hole of $100 \mathrm{~mm}$ in diameter for water drainage. Inside the casing a round tank was placed with an internal diameter of $0.536 \mathrm{~m}$ and a height of 312.5 $\mathrm{mm}$, rigidly secured to the bottom of the casing. Water was supplied into the tank through the tube with the holes of $5 \mathrm{~mm}$ in diameter, arranged along the axis at the sides of the tube; wherein 24 openings on one and the other half of the tube were arranged on its opposite sides. Water was supplied to the tube from the pipeline. The faucet was provided to control water flow. Before the experiment the tube was secured with screws at $100 \mathrm{~mm}$ from the top edge of the tank. All main parts of the hydraulic tray were made of Plexiglas.

Sand of medium size grading, illustrated in Figure 1 was used in the experiments. Sand humidity was $2.5 \%$.

The experiments were carried out to compare dumping sand into the water flow as well as into "still water". The general view of the laboratory setup is shown in Figure 2. 


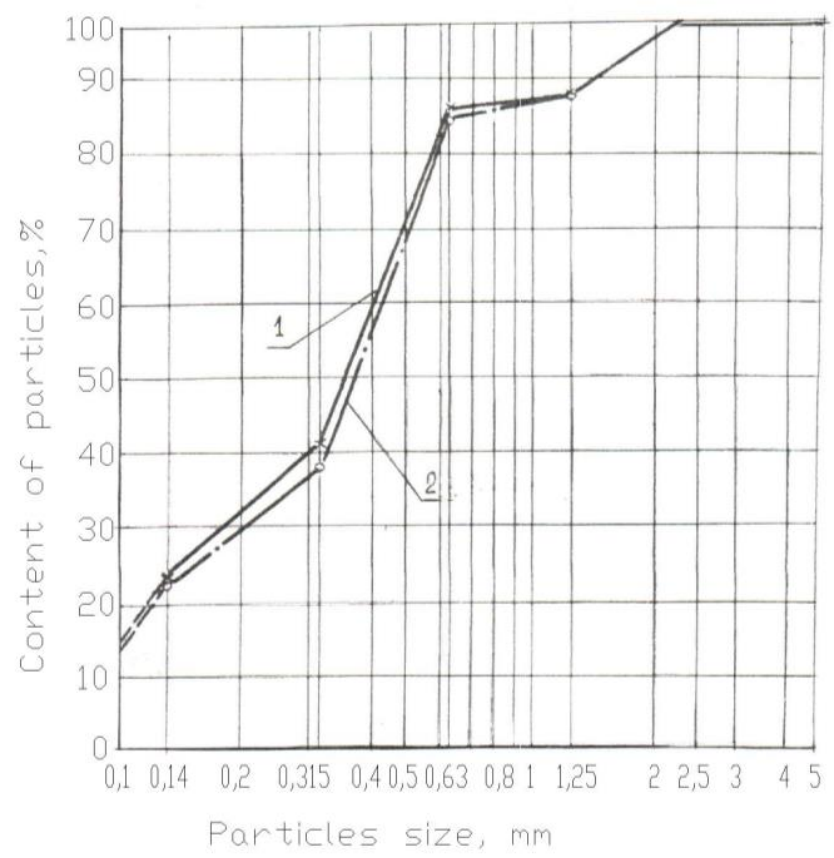

Fig. 1. Size distribution of the soil used in the experiments: 1 and 2 - before the experiment and after the experiment respectively.

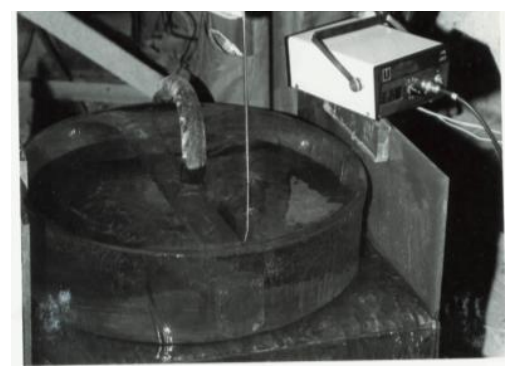

Fig. 2. General view of the laboratory setup during measuring water flow rate.

When dumping into the "still water" the tank was filled with water up to the top edges before the experiment. In the experiment with "running water" water was supplied continuously from the pipeline at a maximum flow rate. In the experiments with "running water" the sand was taken out of the tank into the tray for drying and particle size distribution. This was done to determine the percentage of particles carryover and the diameter of entrained particles. The flow rate at different points and planes of the tank were also measured in the experiments. The measurements were carried out in the empty tank with running water using turntables with the rate measuring range from $3 \mathrm{~cm} / \mathrm{s}$ to $80 \mathrm{~cm} / \mathrm{s}$. The water flow rate measurements obtained in the laboratory experiments did not significantly exceed the calculated values.

\section{Results and analysis}

In the course of dumping sandy soil into "still water" it was found that settling density was $\rho_{\mathrm{sk}}=1,53-1,56 \mathrm{~g} / \mathrm{cm}^{3}$. The density range is indicated for all data obtained in the experiments. 
The density of soil settling can be calculated by the relative density $I_{D}$.

The determined extremum values of the density of dry soil taken for the experiments were the following: the extremum value of loose soil settling density $\rho_{\text {sk }}$ loose $=1,46-1,59 \mathrm{~g} / \mathrm{cm}^{3}$ and the one of dense soil settling $\rho_{\mathrm{sk}}$ dence $=1.74-1.78 \mathrm{~g} / \mathrm{cm}^{3}$.

Thus, the data obtained by dumping sand into "still water" at a given intensity of dumping proved that sand packing density did not exceed $\mathrm{I}_{\mathrm{D}}=0.1$.

This is consistent with the experimental data.

In the experiments with the "water flow" the density of the foundation was the following: $\rho_{\mathrm{sk}}=1,62-1,68 \mathrm{~g} / \mathrm{cm}^{3}$.

Taking into account the percentage of the particles carryover determined by the results of sand particle size distribution before and after the experiment (Fig. 1) that does not exceed 2.5-3\% for the particle size less than $0.315 \mathrm{~mm}$, we have the values of packing density of sandy soil foundation into the water flow as $\rho_{\mathrm{sk}}=1,58-1,63 \mathrm{~g} / \mathrm{cm}^{3}$.

The comparison of the results obtained by dumping sandy soil into "water flow" with the specified parameters and the above-mentioned data on the settling density of soil into "still water" indicates that there is a slight increase in the density of foundation soil settling during dumping into water flow. Sandy soil packing density can increase up to the average density level, which can be acceptable for several types of soil structure foundations.

In addition to the experiments, it is important in the near future to examine the possibility of creating a full-scale installation setup generating movement and fluctuations of water flow during dumping and hydraulic deposition of the foundation soil, i.e. self-oscillation, etc, proposed by I. I. Blehman [18-20].

\section{Conclusions}

Based on the data provided by other researchers and experiments conducted by the author, we can make the following conclusions:

1. The movement of sandy soil particles in a moving water flow with above water inwashing, dumping and underwater inwashing increases the density of compaction of sandy soils of the foundation. In principle, an increase can be reached in compaction of sandy soil density to an average (or maybe even high) level, which can be quite acceptable for the foundations of some types of soil structures.

2. These data were confirmed in the experiments carried out by the author on the dumping of medium size sand in a moving water flow with an average horizontal speed of about 10 $15 \mathrm{~cm} / \mathrm{s}$.

3. In addition, in the near future, it is necessary to consider the possibility of creating fullscale installations that can cause movement and fluctuations of water flow when foundation soil is dumped and inwashed.

4. According to the field studies and practical experience of the author, in all cases , dynamic compaction by various methods can increase the relative density of sands to a high level $I_{\mathrm{D}} \geq 0,6$, which guarantees the stability of the structure of sandy foundation soil for the majority of dynamic and, especially, static effects. In this case, water-saturated sandy soils react weakly even to an explosion.

\section{References}

1. V.A. Florin, P.L Ivanov, Proc. of the Y Intern. Conf. on S. Mech. and Found. 1, 182-186 (1961)

2. P.L. Ivanov, Proc. of the YI Intern. Conf. on S. Mech. and Found. 3, 352-354 (1966) 
3. P.L. Ivanov, N.D. Krasnikov, YI Simp. on Earthq. Eng. 1, 151-156 (1978)

4. P.L. Ivanov, Intern. Conf. on Comp. 1, 331-337 (1980)

5. O.P. Minaev, J. S. Mech. and Found. 30 (2), 53-56 (1993)

6. P. Minaev, J. S. Mech. and Found. 39 (6), 208-213 (2002)

7. P. Minaev, J. S. Mech. and Found. 40 (6), 220-222 (2003)

8. P. Minaev, J. S. Mech. and Found. 48 (5), 190-195(2011)

9. P. Minaev, J. S. Mech. and Found. 50 (6), 251-254(2014)

10. J. Chu, S. Varaksin, U. Klotz, P. Menge, P. Proc, 17 Intern. Conf. on S. Mech. and Geot. TC17, 3006-3035 (2009)

11. B. Hamidi, K. Yee, S. Varaksin, H. Nikraz, L. Wong, 20th Intern. Offsh. and Pol. Eng. Conf., 848-853 (2010)

12. B. Hamidi, S. Varaksin, H. Nikras, Intern. Conf. on Adv. in Geot. Eng. (ICAGE), 221226 (2011)

13. S. Varaksin, B. Hamidi, 18th Intern.Conf. on S. Mech. and Geot. W. (18th ICSMGE) ISP6, 356-363 (2013)

14. P.L. Ivanov, Compaction of cohesionless soils explosions (Nedra, Moscow, 1983)

15. O.P. Minaev, J. S. Mech. and Found. Eng. 31 (4), 141-144 (1994)

16. D.L. Melamut, Hydromechanization in irrigation and agricultural construction (Stroyizdat, Moscow, 1967)

17. A.M. Kurganov, B.F. Fedorov, Hydraulic calculations of water and wastewater systems (Stroyizdat, Moscow, 1986)

18. I. Blekhman, Theory of vibration processes and devices. Vibration mechanics and equipment (Ore and Metals, St. Petersburg, 2013)

19. I.I. Blekhman, J. S. and Vibr. 1-2, 469-470 (2009)

20. V.A. Golovanevskiy, V.A. Arsentev, I.I. Blekhman, V.B. Vasilkov, V.B. Azbel, K.S. Yakimova, J. Min. Proc. 34, 79-85 (2011) 